\title{
Incidence and Clinical Profiles of COVID-19 In Patients with Gynecological Surgery. A Single Center Descriptive Study from Spain
}

\author{
Reyes Oliver ${ }^{1,2}$, Gregorio Lopez-Gonzalez ${ }^{1,2,3}$, Alberto Galindo ${ }^{1,2,3}$, Carmen Alvarez-Conejo ${ }^{1}$, Sofia Aragon- \\ Sanchez ${ }^{1,2}$, Maria Consuelo Sanz-Ferrandez ${ }^{1,2}$ and Alvaro Tejerizo- García ${ }^{1,2,3 *}$ \\ ${ }^{1}$ Department of Obstetrics and Gynecology, Hospital Universitario 12 de Octubre. Madrid, Spain \\ ${ }^{2}$ Instituto de Investigación Hospital 12 de Octubre (imas12), Universidad Complutense de Madrid, Spain
}

${ }^{3}$ Universidad Complutense de Madrid, Spain

*Corresponding author: Alvaro Tejerizo García, Department of Obstetrics and Gynecology, Hospital Universitario 12 de Octubre. Avda, Córdoba s/n. Madrid 28041, Spain.

Received Date: November 02, 2020

Published Date: November 23, 2020

\section{Abstract}

Background: The inflammatory reaction after a surgical intervention could exacerbate the course of the COVID-19. We aim to determine the rate of COVID-19 and its complications among gynecological surgeries in the context of different measures taken during the pandemic period in our department.

Methods: A retrospective longitudinal observational study was conducted. Clinical records of patients who underwent gynecological surgery from March 1st to April 10th, 2020 were reviewed. During this period, three different approaches were made: first phase, without any screening or surgical restrictions; second phase, with presurgical epidemiological screening using a specific questionnaire; and third phase, also with presurgical SARS-COV-19 RT-PCR. During the second and third phases the surgical activity and complexity were restricted, and different workflows were established for patient with suspected/confirmed infection. After hospital discharge, telephone follow-up was performed and screening for COVID-19 was carried out. Complications from the disease were analyzed.

Results: Of the 118 patients that underwent gynecological surgeries, 10 (8.5\%) were perioperatively diagnosed with COVID-19. Of these patients, 8 (80\%) were not pre-surgical screened for SARS-CoV-2 infection, neither clinical nor with RT-PCR. The other 2 (20\%) were preoperative screened with RT-PCR, one of them with a positive test result. Screening false negative rate was $0.8 \%$. No postoperative complications derived from COVID-19 were observed.

Conclusions: The establishment of different surgical workflows, the reduction of surgical complexity, and the use of a pre-surgical screening to detect patient at SARS-CoV-2 infection risk, could reduce the postoperative complications derived from that infection and improve surgical outcomes.

Keywords: COVID-19; SARS-cov-2; Surgery; Gynecology

Abbreviations: COVID-19: Coronavirus Disease; SARS-CoV-2: Severe Acute Respiratory Syndrome Coronavirus 2; R0: Basic Reproductive Number; ARDS: Acute Respiratory Distress Syndrome; ICU: Intensive Care Unit; RT-PCR: Quantitative RNA by Reverse Transcription Polymerase

Chain Reaction; IQR: Interquartile Range

\section{Background}

At the end of 2019, a novel coronavirus disease (COVID-19) was identified as the cause of a cluster of pneumonia cases in Wuhan, a

city in the Hubei Province of China. The virus that causes COVID-19 was designated severe acute respiratory syndrome coronavirus 2 
(SARS-CoV-2). It rapidly spread, resulting in an increasing number of cases in other countries throughout the world. Since the first report of cases from Wuhan, more than 3,267,184 cases worldwide and 215,216 in Spain have been reported [1]. Madrid is one of the main focuses of the epidemic in Spain, with more than 62,205 cases reported until May 2020 [2]. The rapid increase in the number of people infected in a short period of time is mainly due to the highspeed transmission of the virus, with a calculated basic reproductive number (R0) of 2-3 [3].

The incubation period for COVID-19 is thought to be within 14 days, with the majority of cases occurring approximately four to five days following exposure [4-5]. The most common manifestations of COVID-19 include fever, dry cough, dyspnea, myalgia, asthenia, ageusia and anosmia. Most infections are not severe [6-8]. However, complications as acute respiratory distress syndrome [ARDS], arrhythmia, shock, acute cardiac injury, secondary infection, acute kidney injury and death may occur in severe cases [6, 9-11]. This emergency situation has forced health systems around the world to centralize their efforts in eradicating the disease and taking care of the infected people. Consequently, hospitals have become COVID centres and have had to modify their daily activities, including surgical ones.

Currently, the data on the clinical characteristics and outcomes of patients with SARS-CoV-2 infection or COVID-19 undergoing surgeries are rare [11,12]. However, it is postulated that the inflammatory reaction that occurs after a surgical intervention could exacerbate the course of the disease in these patients, conditioning the development of severe complications. In this study we aim to determine the rate of COVID-19 among gynecological surgeries, both prior and posterior to surgery, as well as the patient characteristics and the rate of postoperative complications derived from SARS-CoV-2 infection in the context of different measures taken during the pandemic period in our department.

\section{Methods}

\section{Study design}

A retrospective longitudinal observational study was conducted at Hospital Universitario 12 de Octubre in Madrid, one of the most affected areas of COVID-19 pandemic in Spain. This study was reviewed and approved by our hospital's Ethics Committee. Oral consent was obtained from the patients.
We retrospectively reviewed clinical records of patients who underwent elective or urgent surgery from March 1st to April 10th, 2020. After hospital discharge, telephone follow-up was performed. Screening for symptoms associated with SARS-Cov-2 infection was carried out, and secondary complications from the disease were collected. Pregnant women and those in which postoperative telephone follow-up was not possible were excluded. Telephone calls were performed at least ten days after hospital discharge. Due to the state of alarm and the limitation of mobility of the population and assessing the risk / benefit of attending a hospital center, verbal consent was obtained during the phone call. The clinical outcomes of these patients were monitored up to April 20th, 2020, the final date of follow-up.

Information included demographic characteristics, exposure history, underlying comorbidities, chest CT image, surgical type, surgical time, signs and symptoms, time from surgery to first symptoms, COVID-19 onset and intensive care unit (ICU) admission, treatments, complications, and prognosis. The time of COVID-19 onset was defined as the date when the first sign or symptom was noticed.

\section{Surgical workflow protocols}

From the detection of the first confirmed case of COVID-19 in Spain, on February 24th, 2020, up until now, the surgical management of gynecological patients has been modified in our department, and three different phases can be distinguished:

- $\quad$ First phase, before lockdown was established (from March 1st to March 13th, 2020): all elective or urgent surgery were performed according to the usual criteria without any type of SARS-Cov-2 screening or restriction, with approximately six to ten elective surgical interventions per day.

- $\quad$ Second phase, after establishing the lockdown by the Spanish's Government (from March 14th to March 30th): based on both national and international recommendations and aiming to optimize health resources, surgical activity was restricted to urgent and non-delayable oncological conditions (Figure 1) [13,14]. Clinical and epidemiological screening for SARS-Cov-2 infection was performed in all patients using a specific questionnaire. All elective surgeries in patients clinically suspected of SARS-Cov-2 infection were cancelled (Table 1).

Table 1: Pre- surgical screening questionnaire of COVID-19 symptoms.

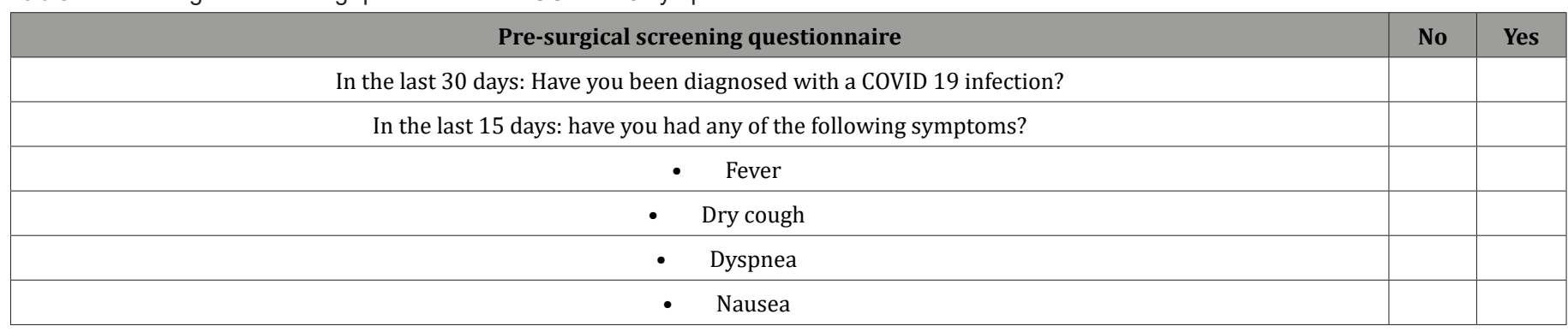




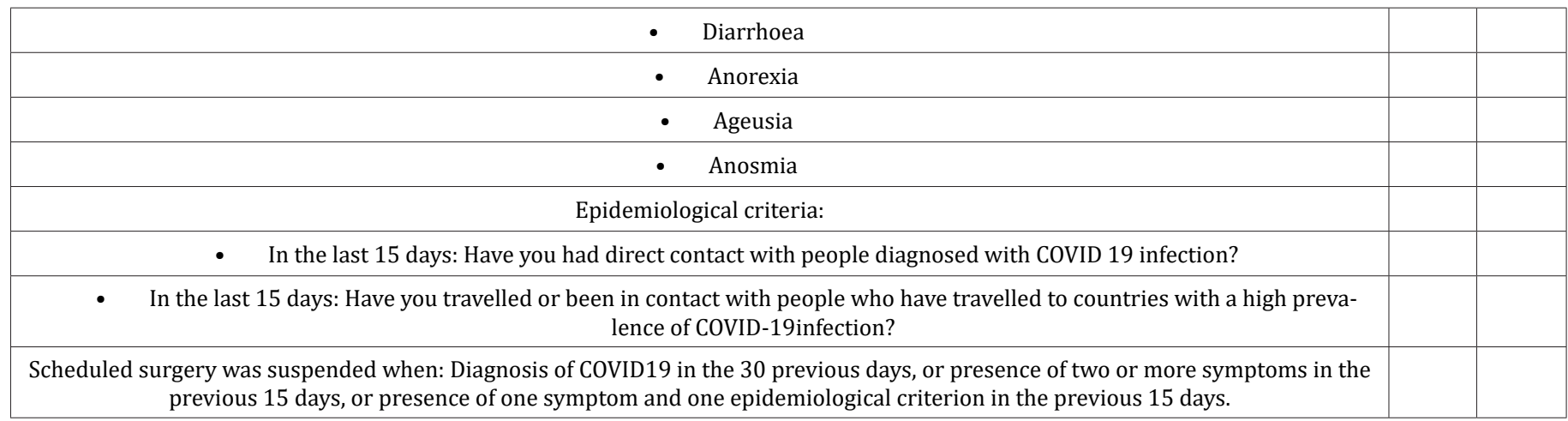

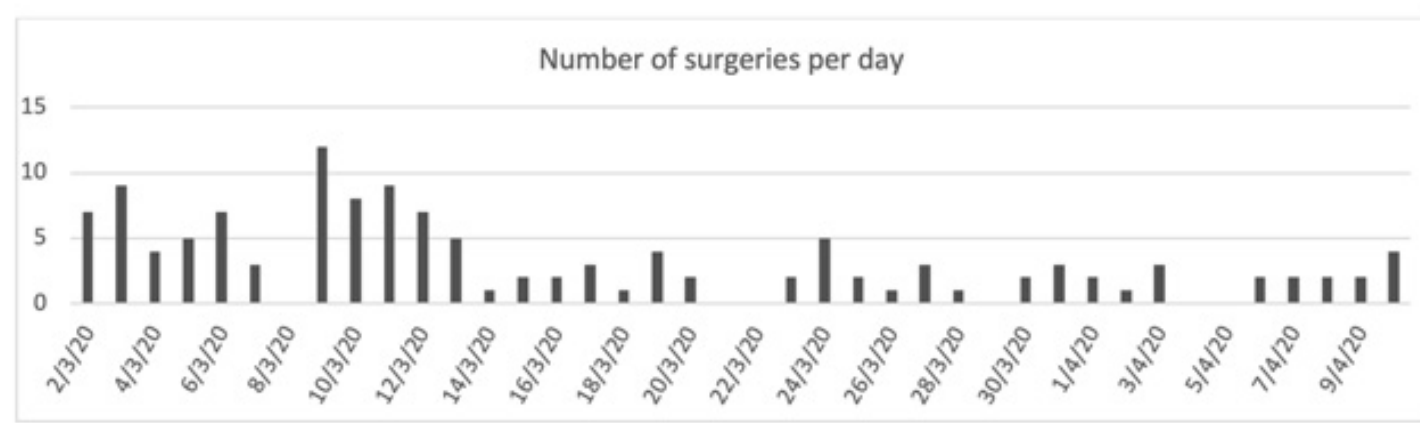

Figure 1: Surgeries in the Department of Obstetrics and Gynecology (Hospital Universitario 12 de Octubre).

Two different areas and independent circuits were established with the aim to separate the suspected/confirmed COVID patients from the asymptomatic/negative ones: an independent operating room and a separate postsurgical stay area (different post-anesthesia care units, and hospitalization) were set for COVID 19 patients. Both the complexity of oncological surgeries and hospital stay were reduced as much as possible.

- $\quad$ Third phase, starting March 30th, 2020, a pre-surgical microbiological screening with RTS-quantitative SARS- CoV-2 PCR was added to previous protocols in all patients. All elective surgeries in patients with a preoperative positive test were cancelled.

\section{Definitions}

The patients included in this study underwent various gynecological surgical procedures, that were categorized into four levels based on the length of surgery and the need of blood transfusion: 1. Level 1 (low), less than 45 minute procedure without needing blood transfusion; 2. Level 2 (moderate), 45 minutes to 2 hours procedure without needing blood transfusion; Level 3 (major), 2 to 6 hours procedure or the one that requires blood transfusion; 4. Level 4 (major complex), more than 6 hours of surgery or the one that requires polytransfusion.

Surgical complications were ranked following the CalvienDindo classification system, that consist of 5 grades [15]. Grade I include any deviation from the normal postoperative course without the need for pharmacological treatment (with the exception of analgesics, antipyretics, antiemetics, diuretics, electrolytes, and physiotherapy) or surgical, endoscopic, and radiological interventions. Grade II complications involves those that require pharmacological treatment different from that allowed for grade I, with blood transfusion and total parenteral nutrition also included. Complications requiring surgical, endoscopic or radiological intervention are considered grade III. Grade IV is defined as lifethreatening complications with the need for intermediate care or ICU management. Finally, grade $\mathrm{V}$ includes the death of a patient due to a complication.

Diagnosis of postoperative SARS-Cov-2 infection was defined as a laboratory confirmation of SAR-CoV 2 by quantitative RNA by reverse transcription polymerase chain reaction (RT-PCR) on samples from the respiratory tract. In absence of confirmation test, both the presence of two or more compatible symptoms and the presence of one symptom with an epidemiological criterion (contact with a confirmed/suspected case during the 15 days prior to the onset of symptoms) were also considered as SARS-COV-2 infection.

\section{Statistical analysis}

Categorical variables were expressed as relative and absolute frequencies. Continuous variables were presented as median with interquartile range (IQR). Categorical variables were compared by Fisher's exact test. A two-sided of less than 0.05 was considered statistically significant. All statistical analyses were performed with the Stata/IC 14.1 software. 


\section{Results}

Gynecological surgery was indicated in a total of 135 patients during the above-mentioned period. However, it was cancelled in five because SARS-Cov-2 infection was diagnosed before surgery: three patients had two or more compatible symptoms when the presurgical questionnaire was performed, and two asymptomatic patients had a positive SARS-CoV-2-quantitative RT-PCR presurgical result. Finally, a total of 130 patients underwent surgery. Of them, 118 were included for analysis, after excluding seven patients who were lost to follow-up and five women pregnant at the time of

Table 2: Baseline characteristics of operative patients.

\begin{tabular}{|c|c|}
\hline No (\%) \\
\hline Age (years), median (IQR) & $49.8(36.7-60.6)$ \\
\hline Any comorbidity & $44(37.3)$ \\
\hline Hypertension & $30(25.4)$ \\
\hline Diabetes & $11(9.3)$ \\
\hline Overweight (25 $\leq$ BMI $\leq 30)$ & $45(38.8)$ \\
\hline Obesity (BMI> 30) & $25(21.6)$ \\
\hline Immunosuppression & $6(5.0)$ \\
\hline Cardiovascular disease & $14(11.9)$ \\
\hline Chronic Kidney disease & $1(0.85)$ \\
\hline Asthma & $6(5.1)$ \\
\hline Chronic Obstructive pulmonary disease & $3(2.5)$ \\
\hline Malignancy & $51(43.2)$ \\
\hline Neoadjuvant chemotherapy & $15(12.7)$ \\
\hline
\end{tabular}

Abbreviations: IQR: Interquartile range; BMI: Body Mass Index; ASA: American Society of Anesthesiologists

Table 3: Types of surgery and postoperative complications.

\begin{tabular}{|c|c|}
\hline & No (\%) \\
\hline Time of admission to surgery & $0.9(0.0-1.0)$ \\
\hline Time of surgery to discharge & $1.6(0.0-3.0)$ \\
\hline Time of admission to discharge & $2.6(1.0-4.0)$ \\
\hline \multicolumn{2}{|c|}{ Surgical procedures } \\
\hline Curettage & $13(11.1)$ \\
\hline Breast lumpectomy +/- SLNB & $29(24.6)$ \\
\hline Mastectomy +/- SLNB & $9(7.6)$ \\
\hline Adnexal surgery & $15(12.7)$ \\
\hline Hysterectomy +/- Bilateral adnexectomy +/- SLNB & $18(15.3)$ \\
\hline Radical hysterectomy +Bilateral adnexectomy +SLNB & $1(0.9)$ \\
\hline Cervical conization & $3(2.6)$ \\
\hline Endometrial Polypectomy & $4(3.4)$ \\
\hline Myomectomy & $4(3.4)$ \\
\hline Sub urethral bands & $3(2.6)$ \\
\hline Diagnostic laparoscopy & $10(8.6)$ \\
\hline Others & $9(7.7)$ \\
\hline \multicolumn{2}{|c|}{ Approach } \\
\hline Vaginal & $32(27.1)$ \\
\hline
\end{tabular}

The baseline characteristics of the patients and types of surgery and postoperative complications are shown in Tables 2 and 3. The median time from admission to surgery and to discharge were 0.9 (0.0-1.0) days and 2.6 (1.0-4.0) days, respectively. Most patients (86.4\%) had no postoperative complications; among those who had any adverse outcome, $62.5 \%$ and $37.5 \%$ were mild (grade 1) and moderate (grade 2) complications, respectively. No major postoperative complications were observed. 


\begin{tabular}{|c|c|}
\hline Breast & $38(32.2)$ \\
\hline Laparoscopy & $27(22.9)$ \\
\hline Laparotomy & $17(14.4)$ \\
\hline Hysteroscopy & $4(3.4)$ \\
\hline \multicolumn{2}{|c|}{ Anaesthesia } \\
\hline General & $79(67.0)$ \\
\hline General + Regional & $35(29.7)$ \\
\hline Epidural & $2(1.7)$ \\
\hline Intradural & $2(1.7)$ \\
\hline Surgical time (minutes) & $125.3(57.0-155.0)$ \\
\hline \multicolumn{2}{|c|}{ Surgical risk category } \\
\hline Level 1- Low & $25(21.2)$ \\
\hline Level 2- Moderate & $9(7.6)$ \\
\hline Level 3- Major & $81(68.6)$ \\
\hline Level 4- Major complex & $3(2.5)$ \\
\hline \multicolumn{2}{|c|}{ Postoperative complications } \\
\hline None & $102(86.4)$ \\
\hline Grade 1 & $10(8.5)$ \\
\hline Grade 2 & $6(5.1)$ \\
\hline Grade 3 & $0(0.0)$ \\
\hline Grade 4 & $0(0.0)$ \\
\hline Grade 5 & $0(0.0)$ \\
\hline
\end{tabular}

Abbreviations: SLNB: Sentinel Lymph Node Biopsy

Table 4: Pre-operative screening and post-operative diagnosis of SARS-COv-2.

\begin{tabular}{|c|c|}
\hline & No (\%) \\
\hline Pre-surgical screening of SARS-COv-2 & $71(60.2)$ \\
\hline None (First phase) & $26(22.0)$ \\
\hline Questionnaire (Second phase) & $21(17.8)$ \\
\hline Questionnaire + RTS-quantitative SARS-CoV 2 PCR (Third phase) & $10(8.5)$ \\
\hline Peri-operative diagnosis of SARS-COV-2 infection & $8(6.8)$ \\
\hline Patients without presurgical screening & $2(1.7)$ \\
\hline
\end{tabular}

Table 5: Clinical characteristics of ten patients suspected COVID-19 infection.

\begin{tabular}{|c|c|c|c|c|c|c|c|c|c|c|c|}
\hline & Patient 1 & Patient 2 & Patient 3 & Patient 4 & Patient 5 & Patient 6 & Patient 7 & Patient 8 & Patient 9 & $\begin{array}{l}\text { Patient } \\
10\end{array}$ & No (\%) \\
\hline Age, years & 55 & 44 & 79 & 43 & 25 & 48 & 39 & 55 & 49 & 48 & $\begin{array}{c}48.8 \\
(43.1-48.3)\end{array}$ \\
\hline \multicolumn{12}{|c|}{ Comorbidities } \\
\hline Hypertension & No & No & Yes & No & No & Yes & No & No & No & Yes & $3(30.0)$ \\
\hline $\begin{array}{c}\text { Cardiovascular } \\
\text { disease }\end{array}$ & No & No & No & No & No & No & No & No & No & No & $0(0.0)$ \\
\hline Diabetes & No & No & No & No & No & No & No & No & No & No & $0(0.0)$ \\
\hline Asthma & No & No & No & No & No & No & No & No & No & No & $0(0.0)$ \\
\hline COPD & No & No & No & No & No & No & No & No & No & No & $0(0.0)$ \\
\hline CKD & No & No & No & No & No & No & No & No & No & No & $0(0.0)$ \\
\hline Malignancy & Yes & No & No & No & No & No & No & No & No & Yes & $2(20.0)$ \\
\hline
\end{tabular}




\begin{tabular}{|c|c|c|c|c|c|c|c|c|c|c|c|}
\hline Type of Surgery & $\begin{array}{c}\text { Excision } \\
\text { of breast } \\
\text { mass }\end{array}$ & $\begin{array}{l}\text { Hysterec- } \\
\text { tomy with } \\
\text { deep endo- } \\
\text { metriosis }\end{array}$ & $\begin{array}{l}\text { Pelvic } \\
\text { Organ } \\
\text { prolapse } \\
\text { correc- } \\
\text { tion }\end{array}$ & $\begin{array}{l}\text { Abdom- } \\
\text { inal wall } \\
\text { endome- } \\
\text { triotic } \\
\text { nodule } \\
\text { resection }\end{array}$ & $\begin{array}{l}\text { Uterine } \\
\text { Curettage }\end{array}$ & $\begin{array}{l}\text { Correc- } \\
\text { tion of } \\
\text { labia } \\
\text { minora } \\
\text { hypertro- } \\
\text { phy }\end{array}$ & $\begin{array}{l}\text { Cervical } \\
\text { conization }\end{array}$ & $\begin{array}{l}\text { Hysteros- } \\
\text { copy } \\
\text { polypec- } \\
\text { tomy }\end{array}$ & $\begin{array}{l}\text { Myomec- } \\
\text { tomy }\end{array}$ & $\begin{array}{l}\text { Unilateral } \\
\text { mastecto- } \\
\text { my }\end{array}$ & \\
\hline $\begin{array}{l}\text { Surgical difficulty } \\
\text { category }\end{array}$ & Level 3 & Level 3 & Level 3 & Level 3 & Level 1 & Level 2 & Level 1 & Level 3 & Level 3 & Level 3 & - \\
\hline Surgical time, min & 100 & 297 & 75 & 105 & 22 & 57 & 30 & 120 & 75 & 175 & $\begin{array}{c}105.6 \\
(57.0-120.0)\end{array}$ \\
\hline Type of anaesthesia & General & General & General & General & General & General & General & General & $\begin{array}{l}\text { Intradu- } \\
\text { ral }\end{array}$ & $\begin{array}{c}\text { General + } \\
\text { Regional }\end{array}$ & - \\
\hline $\begin{array}{c}\text { Pre-Surgical } \\
\text { questionnaire of } \\
\text { COVID-19 Symp- } \\
\text { toms }\end{array}$ & NP & $\mathrm{NP}$ & NP & NP & NP & NP & $\mathrm{NP}$ & NP & Positive & Negative & - \\
\hline $\begin{array}{c}\text { Pre-Surgical test } \\
\text { (SARS-CoV-2- quan- } \\
\text { titative RT-PCR) }\end{array}$ & NP & $\mathrm{NP}$ & NP & NP & NP & NP & NP & NP & Positive & Negative & - \\
\hline $\begin{array}{l}\text { Hospitalization } \\
\text { time, days }\end{array}$ & 5 & 4 & 3 & 3 & 2 & 2 & 1 & 1 & 1 & 4 & $2.6(1.0-4.0)$ \\
\hline \multicolumn{12}{|c|}{ Signs and symptoms } \\
\hline Fever & Yes & No & No & Yes & No & Yes & Yes & No & Yes & Yes & $6(60.0)$ \\
\hline Asthenia & No & Yes & No & No & Yes & No & No & Yes & No & No & $3(30.0)$ \\
\hline Dry Cough & No & Yes & No & Yes & No & Yes & Yes & No & Yes & No & $5(50.0)$ \\
\hline Dyspnea & No & No & No & No & Yes & No & No & No & No & No & $1(10.0)$ \\
\hline Myalgia & No & Yes & No & No & Yes & No & No & Yes & No & No & $3(30.0)$ \\
\hline Headache & Yes & Yes & No & No & Yes & Yes & No & No & Yes & No & $5(50.0)$ \\
\hline Anorexia & No & Yes & No & No & Yes & No & Yes & No & No & No & $3(30.0)$ \\
\hline Gastrointestinal & No & Yes & Yes & No & No & No & No & No & No & No & $2(20.0)$ \\
\hline Ageusia/Anosmia & No & No & Yes & No & Yes & Yes & No & No & Yes & No & $4(40.0)$ \\
\hline $\begin{array}{l}\text { First symptom or } \\
\text { sign }\end{array}$ & Fever & Cough & $\begin{array}{c}\text { Ageusia } \\
\text { Anosmia }\end{array}$ & Cough & Headache & Fever & Fever & Myalgia & Cough & Fever & \\
\hline $\begin{array}{c}\text { Time from Surgery } \\
\text { to onset symptoms, } \\
\text { days }\end{array}$ & 12 & 22 & 3 & 15 & 28 & 18 & 2 & 35 & -3 & 2 & $\begin{array}{c}13.6(2.0- \\
22.0)\end{array}$ \\
\hline $\begin{array}{l}\text { Epidemiological } \\
\text { history* }\end{array}$ & Yes & Yes & No & Yes & No & Yes & Yes & Yes & Yes & Yes & $8(80.0)$ \\
\hline $\begin{array}{l}\text { Confirmatory test } \\
\text { (SARS-CoV-2-quan- } \\
\text { titative RT-PCR) }\end{array}$ & Yes & No & No & No & No & No & No & No & Yes & No & \\
\hline Hospitalization & No & No & No & No & No & No & No & No & No & No & $0(0.0)$ \\
\hline \multicolumn{12}{|l|}{ Complications } \\
\hline Respiratory failure & No & No & No & No & No & No & No & No & No & No & $0(0.0)$ \\
\hline ARDS & No & No & No & No & No & No & No & No & No & No & $0(0.0)$ \\
\hline Shock & No & No & No & No & No & No & No & No & No & No & $0(0.0)$ \\
\hline Acute cardiac injury & No & No & No & No & No & No & No & No & No & No & $0(0.0)$ \\
\hline Acute Kidney injury & No & No & No & No & No & No & No & No & No & No & $0(0.0)$ \\
\hline Arrhythmia & No & No & No & No & No & No & No & No & No & No & $0(0.0)$ \\
\hline Death & No & No & No & No & No & No & No & No & No & No & $0(0.0)$ \\
\hline
\end{tabular}

Data of pre-operative screening and post-operative diagnosis of SARS-COv-2 are shown in Table 4. In summary, $10(8.5 \%)$ of the 118 operated patients were perioperatively diagnosed with
COVID-19. Eight $(80 \%)$ of these patients underwent surgery before the lockdown, in the first period, without performing any pre-surgical screening of SARS-COV-2, neither questionnaire nor 
RT-PCR, and the diagnosis was made after surgery. The other two patients $(20 \%)$ were preoperative screened with SARS-COV-2 quantitative PCR (third period), one of them with a positive test result. Eight of the seventy-one (11.3\%) patients without presurgical screening and two of the forty-seven (4.3\%) patients with presurgical screening were diagnosed with COVID-19 ( $p=0.1)$. The patient with confirmed pre-surgical COVID-19 infection (Table 5, patient 9, questionnaire and RTS-quantitative SARS-COV-2 PCR positives) underwent surgery despite it, because of prolapse of a submucosal leiomyoma through the cervix and profuse vaginal bleeding. A vaginal myomectomy was performed.

The baseline profiles of the ten patients are shown in Table 5. Three $(30.0 \%)$ had one or more comorbidities, and two $(20.0 \%)$ had a current diagnosis of gynecological cancer. The median time from surgery to the onset of COVID-19 was 13.6 (IRQ 2.0-22.0) days. Excluding the symptomatic patient at the time of surgery (patient 9), in three patients (33.3\%) the first symptom of the disease occurred during the first four days after surgery, in one $(11.1 \%)$ between days five to fourteen, and in five patients (55.6\%) from day fifteen onwards. Seven (70\%) patients had had contact outside the hospital with a confirmed/suspected case during the 15 days prior to the onset of symptoms. None of the 10 patients needed hospital admission because of COVID-19 infection itself. Only one patient presented mild unilateral pneumonia that resolved without complications with outpatient treatment.

\section{Discussion}

On February 28th, 2020 the first two cases of COVID-19 infection were detected in the Region of Madrid (Spain), with a subsequent rapid spread, reaching 44,783 positive cases on April 10th (Figure 2) [2]. This exponential increase in the number of newly infected overloaded the emergency departments and ICU of the hospitals in Madrid's region. Human and material resources and daily activities were then reorganized to assist the high number of patients.

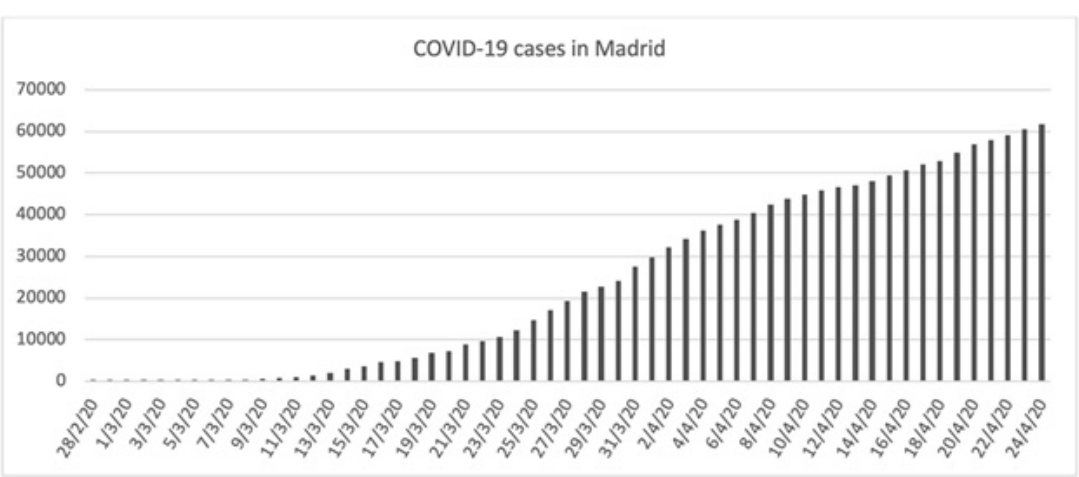

Figure 2: Covid-19 cases in Madrid [2].

Due to the architectural design of our Hospital, the Women's and Children's Care Center are separated from the main Hospital building, where the vast majority of Covid-19 patients were admitted. Therefore, our department was able to continue some surgical activity, even at most saturated hospitalization moments.

The spectrum of SARS-Cov-2 symptomatic infection ranges from mild to critical. Fortunately, most infections are not severe [5-10]. Nevertheless, some authors suggest that surgery may accelerate and exacerbate the course of the infection, given that surgery may cause an immediate impairment of cell-mediated immunity, one of the major mechanisms that bring viral infections under control $[11,12,16,17]$. Lei, et al. published the data of a retrospective cohort of 34 patients underwent elective surgeries, during the incubation period of COVID-19 in four different hospitals of Wuhan, China $44,1 \%$ of the patients needed ICU and the mortality rate was $20,5 \%$ [11]. This proportion of intensive care need is much higher than the reported (range 14-26\%) in hospitalized COVID-19 patients without surgery [18].

In our series, the incidence of patients with COVID-19 after surgery was $8.5 \%$. In contrast with Lei et al. none of the patients presented postoperative complications or required hospital admission due to the SARS-Cov-2 infection. These differences may be explained by different factors. Firstly, is the time from surgery to the onset of symptoms. For Lei, et al. the median time from surgery to the first symptom was 2.0 days whereas in our series it was 13.6 days. The incubation period for COVID-19 is thought to be within 14 days, being in most cases around 4-5 days after exposure [4,5]. However, only $44,4 \%$ of our patients became symptomatic in the first 14 days after surgery, and 33,3\% in the first 5 days. Therefore, most of our patients underwent surgery out of the incubation period and probably they became infected after hospital discharge, minimizing thereby the inflammatory postoperative reaction. Most cases who developed symptoms underwent surgery during the phase 1 (no restrictions). Only two infected patients were operated during established screening periods. One patient who underwent an emergent surgery with a known positive PCR test and another one with a previous negative PCR test that developed symptoms two days after surgery with a final positive PCR test (considered as a false negative). None of the 26 patients operated in the second period (only epidemiologic questionnaire) were infected subsequently. Thus, our false negative rate for these screening 
measures was $0.8 \%$.

Secondly, the type of surgeries included could be another factor. In the study of Lei et al., only three patients withstood gynecological surgery and all of them were radical. In contrast, according to the main scientific gynecological societies $[13,14]$, after the declaration of the state of alarm, the surgical complexity was reduced in our department in order to reduce surgical adverse events and shorten postoperative hospital stay. This approach could have decreased the adverse outcomes in patients who underwent surgery during the incubation period, as well as have minimized the hospital exposure of uninfected patients. In this line, also individual protection measures for health professionals were increased and two different areas and independent workflows were established for suspected/ confirmed COVID-19 patients. All the measures taken during these periods in our department were focused on achieving three objectives: to identify positive COVID-19 patients and place them in isolated areas, to prevent the risk of transmission of the virus to uninfected patients, increase the safety of healthcare personnel and try to minimize possible postoperative complications in COVID-19 positive patients.

Although the overall incidence of patients with COVID-19 after surgery was $8.5 \%$, it was higher in the group of patients in whom no preoperative screening was done $(11.3 \%$ vs. $4.2 \%$; $=0.1)$. In addition, five more elective surgeries were cancelled because of a positive SARS-Cov-2 infection screening, avoiding the possible morbidity/mortality derived from the infection and surgery in these patients.

This data supports the implementation of an early COVID- 19 infection screening to avoid surgical procedures in the incubation period. The diagnosis of COVID-19 is made by detection of SARSCoV-2 RNA by RT-PCR, but in many cases, because of the limited availability of testing and concern for false-negative results, the diagnosis of COVID-19 is made presumptively based on a compatible clinical presentation in the setting of an exposure risk $[19,20]$. Therefore, excluding epidemiological criteria and COVID-19 symptoms before surgery is imperative and it can be sufficient in most cases if the RT-PCR is lacking.

Our study has several limitations. The sample size of the present study is small. Additionally, not all patients with COVID-19 symptoms had a confirmation test, due to the limited understanding of the epidemic situation and the shortage of SARS-CoV-2 kits at that time. Moreover, we have used an internal classification system of the surgical complexity that has not been standardized among centers, that preclude robust comparisons with other series. However, most centers used different combinations of surgical indicators to define the complexity, since it is believed that the more markers are used the better is the definition of the complexity. However, to the best of our knowledge, this is the first retrospective cohort study from a single center describing the incidence and clinical characteristics and outcomes of COVID-19 in patients with gynecological surgery.
Accordingly, both knowledge of the data on the impact of surgery performed during the incubation period of COVID-19 infection and universal pre-operative screening of SARS-Cov-2 infection could reduce the morbidity and mortality of these patients.

\section{Conclusion}

In conclusion, we consider that establishing independent surgical workflows, correctly selecting the type of surgeries to be performed and the use of a pre-surgical screening to detect patients at risk will improve the surgical results in pandemic periods as the one we live in.

\section{Acknowledgement}

Elisa Simon.

\section{Declarations}

\section{Ethics approval and consent to participate}

This study and the informed consent form have been approved by the Research Ethics Committee of the University Hospital 12 de Octubre (Comité Ético Investigacion Clínica con Medicamentos, CEIm Hospital 12 de Octubre) with the reference number: 20/203.

The informed consent was obtained from all the patients and it was verbal because of the state of alarm decreed in Spain, trying to reduce the number of displacements to the Hospital.

\section{Consent for publication}

All authors consent and authorize publication of the article by BMC Surgery.

\section{Availability of data and materials}

The datasets used and/or analysed during the current study are available from the corresponding author on reasonable request.

None of the patients who have been included in this study have participated in other studies carried out by the authors of this article or by any other author.

\section{Authors Contributions}

AT had the idea of the study. AT and RO designed the study. RO have full access to all data in the study and take responsibility for the integrity of the data and the accuracy of the data analysis. RO, CA, CS, SA, GL collected the data. RO performed data analysis. RO and AT drafted the manuscript. RO, CA, CS, SA, GL, AG and AT revised the final manuscript. All authors have read and approved the manuscript.

\section{Conflict of Interest}

The authors declare that they have no conflicts of interest and nothing to disclose.

\section{References}

1. (2020) World Health Organization. Coronavirus disease (COVID-19) Situation report- 103. 
2. (2020) Instituto de salud Carlos III. Situación de COVID en España.

3. Liu Y, Gayle AA, Wilder-Smith A, Rocklöv J (2020) The reproductive number of COVID-19 is higher compared to SARS coronavirus. J Travel Med 27(2): taaa021.

4. Li Q Guan X, Wu P, Wang X, Zhou L, et al. (2020) Early transmission dynamics in Wuhan, China, of Novel Coronavirus-infected pneumonia. N Engl J Med 382: 1199.

5. Chan JF, Yuan S, KOK KH, To K, Chu H, et al. (2020) A familial cluster of pneumonia associated with the 2019 novel coronavirus indicating person-to-person transmission: A study of a family cluster. Lancet 395(10223): 514.

6. Wang D, Hu B, Hu C, Zhu F, Liu X, et al. (2020) Clinical Characteristics of 138 Hospitalized Patients With 2019 Novel Coronavirus-Infected Pneumonia in Wuhan, China. JAMA 323(11): 1061-1069.

7. Lauer SA, Grantz KH, Bi Q, Jones FK, Zheng Q, et al. (2020) The Incubation Period of Coronavirus Disease 2019 (COVID-19) From Publicly Reported Confirmed Cases: Estimation and Application. Ann Intern Med 172((9): 577-582.

8. Bajema KL, Oster AM, McGovern OL, Lindstrom S, Stenger MR, et al. (2020) Persons Evaluated for 2019 Novel Coronavirus - United States, January 2020. MMWR Morb Mortal Wkly Rep 69(6):166-170.

9. Huang C, Wang Y, Li X, R Lili, Z Jianping, et al. (2020) Clinical features of patients infected with 2019 novel coronavirus in Wuhan, China. Lancet 395(10223): 497-506.

10. Chen N, Zhou M, Dong X, Qu J, Gong F, et al. (2020) Epidemiological and clinical characteristics of 99 cases of 2019 novel coronavirus pneumonia in Wuhan, China: a descriptive study. Lancet 395(10223): 507-513.

11. Lei S, Jiang F, Su W, Chen C, Chen J, et al. (2020) Clinical characteristics and outcomes of patients undergoing surgeries during the incubation period of COVID-19 infection. EClinicalMedicine 5: 100331.
12. Chen H, Guo J, Wang C, Luo F, Yu X, et al. (2020) Clinical characteristics and intrauterine vertical transmission potential of COVID-19 infection in nine pregnant women: a retrospective review of medical records. Lancet 395(10226): 809-815.

13. (2020) Spanish Society of Gynecology and Obstetrics. Recommendations of the SEGO Gynecological Oncology and Breast Pathology Section for the management of gynecological tumors during the COVID-19 pandemic.

14. Ramirez PT, Chiva L, Eriksson AGZ, Frumovitz M, Fagotti A, et al. (2020) COVID-19 Global Pandemic: Options for Management of Gynecologic Cancers. Int J Gynecol Cancer 30(5): 561-563.

15. Dindo D, Demartines N, Clavien PA (2004) Classification of surgical complications: a new proposal with evaluation in a cohort of 6336 patients and results of a survey. Ann Surg 240: 205-213.

16. Amodeo G, Bugada D, Franchi S, Moschetti G, Grimaldi S, et al. (2018) Immune function after major surgical interventions: the effect of postoperative pain treatment. J Pain Res 11: 1297-1305.

17. Hermesh T, Moltedo B, López CB, Moran TM (2010) Buying time-the immune system determinants of the incubation period to respiratory viruses. Viruses 2(11): 2541-2558.

18. Richardson S, Hirsch JS, Narasimhan M, Crawford JM, McGinn T, et al. (2020) Presenting Characteristics, Comorbidities, and Outcomes Among 5700 Patients Hospitalized With COVID-19 in the New York City Area. JAMA 323(20): 2052-2059.

19. Fang Y, Zhang H, Xie J, Lin M, Ying L, et al. (2020) Sensitivity of Chest CT for COVID-19: Comparison to RT-PCR. Radiology 296(2): E115-E117.

20. Lee TH, Lin RJ, Lin RTP, Barkham T, Rao P, et al. (2020) Testing for SARSCoV-2: Can We Stop at Two? Clin Infect Dis ciaa459. 